\title{
Experimental time-delayed feedback control with variable and distributed delays
}

\author{
Thomas Jüngling, ${ }^{1, *}$ Aleksandar Gjurchinovski, ${ }^{2}$ and Viktor Urumov ${ }^{3}$ \\ ${ }^{1}$ Institute for Cross-Disciplinary Physics and Complex Systems, University of the Balearic Islands, 07122 Palma de Mallorca, Spain \\ ${ }^{2}$ Institute of Physics, Faculty of Natural Sciences and Mathematics, Saints Cyril and Methodius University, P. O. Box 162, \\ 1000 Skopje, Macedonia \\ ${ }^{3}$ Partenij Zografski 46, 1000 Skopje, Macedonia
}

(Received 1 March 2012; revised manuscript received 10 September 2012; published 24 October 2012)

\begin{abstract}
We report on several improvements of the classical time-delayed feedback control method for stabilization of unstable periodic orbits or steady states. In an electronic circuit experiment, we were able to realize time-varying and distributed delays in the control force leading to successful control for large parameter sets, including large time delays. The presented techniques make advanced use of the natural torsion of the orbits, which is also necessary for the original control method to work.
\end{abstract}

DOI: 10.1103/PhysRevE.86.046213

PACS number(s): $05.45 . \mathrm{Gg}, 02.30 . \mathrm{Ks}$

\section{INTRODUCTION}

The control of chaos has attracted the attention of physicists for over two decades now. The most established method for controlling unstable periodic orbits embedded in a chaotic attractor is the application of a continuous time-delayed feedback, which was first introduced by Pyragas [1]. A dynamical system

$$
\dot{\mathbf{x}}=\mathbf{f}(\mathbf{x})
$$

subjected to Pyragas control becomes

$$
\dot{\mathbf{x}}=\mathbf{F}\left(\mathbf{x}, k\left(\mathbf{x}_{\tau}-\mathbf{x}\right)\right),
$$

where $\mathbf{x}_{\tau} \equiv \mathbf{x}(t-\tau)$, and $k$ is the feedback gain. The control is applied in such a way that $\mathbf{F}(\mathbf{x}, \mathbf{0})=\mathbf{f}(\mathbf{x})$. To control an unstable periodic orbit, the delay $\tau$ is adjusted to match the period of the orbit. When stabilization is achieved, the control signal vanishes by construction, meaning that the controlled orbit remains a solution of the original Eq. (1). This property of the Pyragas method is known as noninvasiveness. For stabilization of unstable steady states, the choice of the delay time $\tau$ is not as restrictive as in the case of unstable periodic orbits, and the interval of $\tau$ for which the control is successful is shown to be system-dependent. Examples of successful control are shown in Fig. 1.

The Pyragas method was successfully implemented in a variety of experimental setups, and an effort has been put forth to generalize or modify the original control scheme in order to improve its performance. An overview of the field is given in [2]. An important generalization of the Pyragas method was proposed by Socolar et al. [3], where the feedback signal was taken in the form of a geometric sum [extended timedelayed feedback control (ETDFC)], or a mean value of a finite number of delay terms [4] [ $N$ time-delayed feedback control (NTDFC)], each using information from many previous states of the system involving integer multiples of the delay time $\tau$. A natural extension of this method was proposed by Ahlborn and Parlitz [5,6] using two or more delayed feedback signals with incommensurate delay times. These multiple delay extensions

\footnotetext{
*thomas@ifisc.uib-csic.es
}

achieve stabilization of unstable states with a higher degree of instability.

In a recent work [7], it was shown that the efficiency of the Pyragas method to control unstable steady states is significantly improved by applying a variable time delay in the original control scheme. We call this method variable-delay feedback control (VDFC). In this case, the modulation of the delay is in a fixed interval around some nominal delay value, and it can be realized in a deterministic or a stochastic way. The essence of the improved control mechanism lies in the delay distribution, which is created by the modulation. It has already been reported that delay distributions in coupled oscillators lead to stabilization of steady states [8-10]. The same mechanism applies to self-feedback on a single oscillator.

In this work, we experimentally investigate the control of steady states and periodic orbits using variable-delay feedback as well as distributed-delay feedback. In Sec. II, the experimental setup is described. The possibilities and constraints for the construction of variable and distributed delays are shown. The relation between modulation and distribution is explained in Sec. III. In Sec. IV, we study three different methods of fixed point control, whereas Sec. V deals with the control of a periodic orbit. Finally, in Sec. VI the control mechanism, which is mostly covered theoretically, is summarized and interpreted. The final section contains our conclusions.

\section{EXPERIMENT}

Our experimental setup consists of a single electronic oscillator subjected to time-delayed feedback. A schematic of the circuit is shown in Fig. 2. This circuit has already been used in different contexts and is described there in detail [11-13]. The equations of motion for the main dynamical components are similar to the Rössler system and read explicitly

$$
\begin{aligned}
& \dot{x}=\omega_{0}(-c x-y-z), \\
& \dot{y}=\omega_{0}[x+(a-c) y], \\
& \dot{z}=\omega_{0}[g(x, z)-\gamma z],
\end{aligned}
$$

where $g(x, z)=b(|w|+w)$ with $w=x+z / 2-z_{\text {thr. The }}$. The variables $x, y, z$ are voltages taken at $X 1, X 2$, and $X 3$, respectively. The time scale of the oscillator is determined 

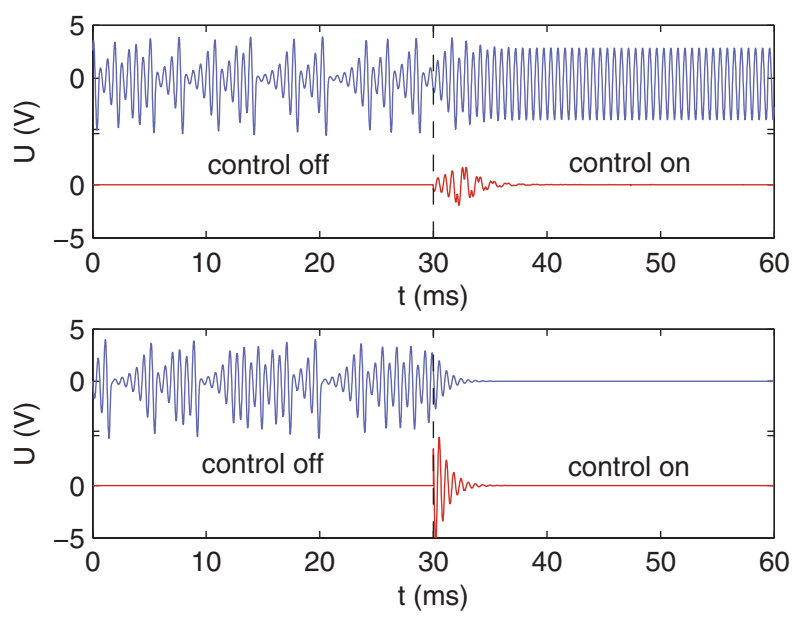

FIG. 1. (Color online) Trajectories from the electronic circuit Fig. 2 at the onset of time-delayed feedback control. Top: Control of a periodic orbit with delay $\tau=T_{p}=0.63 \mathrm{~ms}$. Bottom: Control of a fixed point with delay $\tau=0.32 \mathrm{~ms}$. Blue (upper): Signal $y(t)$ recorded from the oscillator. Red (lower): Control signal $v(t)=$ $y(t-\tau)-y(t)$. Control is switched on at $t=30 \mathrm{~ms}$.

by $\omega_{0}=1 / R_{4} C=10 \mathrm{ks}^{-1}$, so that typical oscillations have a period close to $T_{0}=2 \pi / \omega_{0}=0.63 \mathrm{~ms}$. The parameters $c=0.05, b=3.18, \gamma=2.82$, and $z_{\mathrm{thr}}=3.35 \mathrm{~V}$ are optimized in such a way as to obtain robust dynamics and a welldefined bifurcation structure by variation of the main circuit parameter $a$. Our standard value is $a=0.3$, for which a chaotic

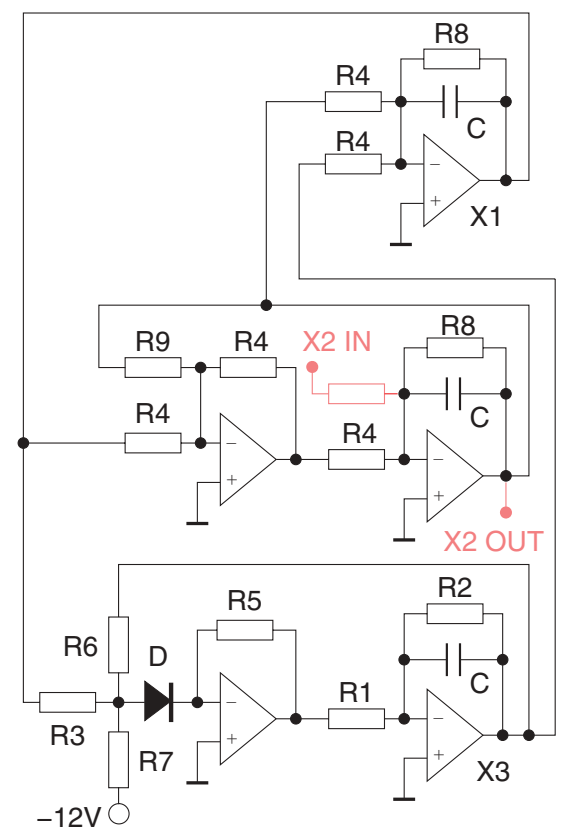

FIG. 2. (Color online) The circuit scheme of the autonomous diode oscillator with inputs and outputs for coupling [light red (gray)]. Values of components: $R_{1}=2.7 \mathrm{k} \Omega, R_{2}=3.6 \mathrm{k} \Omega, R_{3}=$ $7.5 \mathrm{k} \Omega, R_{4}=10 \mathrm{k} \Omega, R_{5}=13 \mathrm{k} \Omega, R_{6}=15 \mathrm{k} \Omega, R_{7}=33 \mathrm{k} \Omega, R_{8}=$ $200 \mathrm{k} \Omega . \quad R_{9}$ is variable $0-50 \mathrm{k} \Omega$ and determines the system parameter $a$ according to $R_{9}=10 \mathrm{k} \Omega / a$ (accuracy of resistors $1 \%$ ). Capacitors: $C=10 \mathrm{nF}$ (accuracy 5\%). Type of diode: 1N4007 from DC Components. Type of operational amplifiers: TL084.

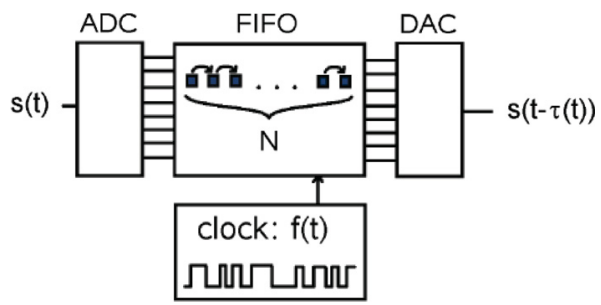

FIG. 3. (Color online) A single delay line with clock frequency modulation.

attractor with a maximum Lyapunov exponent of $\Lambda=0.1 \omega_{0}$ is observed.

Time-delayed feedback is applied to the $y$ component, whose governing equation is thus changed into

$$
\dot{y}=\omega_{0}\left[x+(a-c) y+k\left(y_{\tau}-y\right)\right],
$$

where $y_{\tau}=y(t-\tau)$ is the delayed $y$ signal, and $k$ is the feedback gain parameter. To get a clear time-delayed signal of this component with delay times $\tau$ in the order of milliseconds, we use digital delay lines as shown in Fig. 3. The analog signal in the range from -10 to $+10 \mathrm{~V}$ is first scaled to fit between 0 and $5 \mathrm{~V}$. Then it is discretized by an analog-to-digital converter (ADC) with 8 bits, allowing a resolution of $78 \mathrm{mV}$ in the original voltage scale. Delay is created by storing the signal in a first-in-first-out device (FIFO) with a maximum of $1 \mathrm{kB}$ memory. The device is triggered by an externally prepared write-clock and read-clock, which defines the number $N$ of stored samples of the signal and the rate $f$ by which they are shifted through the register. Finally, the output of the FIFO is converted back by a digital-to-analog converter (DAC) and low-pass filtered to clear the steplike behavior of the signal before rescaling to the original voltage range. The low-pass filter is of second order with a cutoff frequency $f_{\mathrm{lp}}=20 \mathrm{kHz}$. The frequency gap between $f_{\mathrm{lp}}$ and the sampling frequency $f$ is used for the dithering effect by applying a suitable small noise signal, which interpolates between the bits of the ADC. Under optimal constraints, we can effectively reach a resolution of 11 bits instead of 8 bits provided by the devices without dithering. This corresponds to an absolute resolution of $10 \mathrm{mV}$. With the use of the dithering effect, there is a tradeoff between voltage resolution and frequency resolution. The described settings have shown to be optimal for our purposes. We have constructed several such delay lines, which can be adjusted to different delays simultaneously so we can implement a large variety of different feedback signals.

The clock signal for the delay line is created by a function generator type Agilent 33220A. It allows an arbitrary modulation of the clock frequency, which results in a delay time modulation $\tau(t)$. The relation between clock frequency modulation and delay time modulation for the given delay lines can be derived as

$$
N=\int_{t-\tau(t)}^{t} f\left(t^{\prime}\right) d t^{\prime} .
$$

Here $N$ is the buffer size of the FIFO, $f(t)$ is the current clock frequency, and $\tau(t)$ is the current delay time, i.e., if $y(t)$ is the signal at the input of the delay line, then we have at the output $y[t-\tau(t)]$. Equation (5) does not allow for an arbitrary delay time modulation, for instance, we cannot realize a jump in $\tau(t)$. 
For a delay modulation which includes steplike functions $\tau(t)$, we therefore choose to switch between different delay lines. A similar technique is applied in Sec. V, where only discrete values of the delay time are allowed.

\section{MODULATION AND DISTRIBUTION}

In the following, we consider the types of modulation for which we can write

$$
\tau(t)=\tau_{0}+\varepsilon \tilde{\tau}(t),
$$

where $\varepsilon$ is the modulation amplitude and $\tilde{\tau}(t) \in[-1,1]$ is a periodic signal with period $T_{m}=f_{m}^{-1}$. It may also be an irregular bounded signal, for which the power spectrum has at least a lower cutoff frequency $f_{\text {co }}$. In the limit case of a very high modulation frequency $f_{m} \gg f_{0}$ or a very high cutoff frequency $f_{\text {co }} \gg f_{0}$, respectively, the modulated delay is equivalent to a delay distribution $\rho(\tau) . f_{0}=T_{0}^{-1}=1.59 \mathrm{kHz}$ is the natural frequency of the oscillator. The density function $\rho(\theta)$ is defined in such a way that for an increment $d \theta$, the fraction of time that $\tau(t)$ is found to lie between $\theta$ and $\theta+d \theta$ is given by $\rho(\theta) d \theta$. A rigorous result establishing the relation between variable delay and the corresponding delay distribution was reported by Michiels et al. [14]. An example is provided by rectangular (square wave) modulation, in which $\tau(t)$ switches periodically between $\tau_{0}-\varepsilon$ and $\tau_{0}+\varepsilon$, both for the same duration. This leads to a two-peak distribution

$$
\rho(\theta)=\frac{1}{2}\left[\delta\left(\theta-\tau_{0}+\varepsilon\right)+\delta\left(\theta-\tau_{0}-\varepsilon\right)\right] .
$$

Another example is a triangular (sawtooth) modulation. The delay $\tau(t)$ rises and falls linearly between its extreme values, which results in a uniform distribution. It is not affected by the skewness of the sawtooth wave, and we always obtain

$$
\rho(\theta)= \begin{cases}\frac{1}{2 \varepsilon}, & \theta \in\left[\tau_{0}-\varepsilon, \tau_{0}+\varepsilon\right], \\ 0, & \text { otherwise. }\end{cases}
$$

Finally, a sinusoidal modulation leads to

$$
\rho(\theta)=\frac{1}{\pi \sqrt{\varepsilon^{2}-\left(\theta-\tau_{0}\right)^{2}}} .
$$

As we show later, in most cases in which delay time modulation improves stability, the mechanism can essentially be reduced to the delay distribution created by the modulation. However, there are also cases in which a finite modulation frequency plays a role for stabilization. This issue is discussed in Sec. V.

\section{STABILIZATION OF FIXED POINTS}

As we have shown previously in a theoretical work [7], a delay time modulation may enhance the stability properties of a Pyragas-controlled fixed point significantly. We demonstrate this effect in our electronic experiment using different types of delay modulations (distributions). The free-running circuit has an unstable fixed point at the coordinate origin. Equations (3) also have another fixed point solution, but it lies outside the experimental voltage range, so we investigate only the central fixed point $\left(x_{0}, y_{0}, z_{0}\right)=(0,0,0)$. Its stability is determined by the eigenvalues of $\mathbf{D f}(\mathbf{0})$, which denotes the Jacobian of the vector field $\mathbf{f}$ at the fixed point

$$
\mathbf{D f}(\mathbf{0})=\omega_{0}\left(\begin{array}{ccc}
-c & -1 & -1 \\
1 & a-c & 0 \\
0 & 0 & -\gamma
\end{array}\right) .
$$

The eigenvalues of this matrix are $\lambda_{1 / 2}=\omega_{0}\left(\frac{a}{2}-c \pm\right.$ $\left.\sqrt{a^{2} / 4-1}\right)$ and $\lambda_{3}=-\gamma \omega_{0}$. For standard parameters, the first two are a complex conjugate pair with a positive real part, $\lambda_{1 / 2} \approx(0.1 \pm i) \omega_{0}$, and the third one is negative, $\lambda_{3}=$ $-2.82 \omega_{0}$. The fixed point is susceptible to Pyragas control because the imaginary parts of $\lambda_{1 / 2}$ are nonzero, so that the neighborhood of the fixed point undergoes a $2 \pi$ torsion in the time interval $T_{0}=0.63 \mathrm{~ms}$. For the sake of simplicity, we further discuss the stability properties of the fixed point in terms of a normal form

$$
\dot{z}=\lambda_{0} z
$$

with $z \in \mathbb{C}$ and $\lambda_{0}=(a / 2-c)+i$. The fixed point is at the origin $z=0$ and the oscillation period around it is $T_{0}=2 \pi$. The results obtained by the normal form are in very good agreement with all our findings from the full system of differential equations as well as with the experimental outcomes. A Pyragas-type feedback changes Eq. (11) to

$$
\dot{z}=\lambda_{0} z+\kappa\left(z_{\tau}-z\right),
$$

with $z_{\tau} \equiv z(t-\tau)$. To identify the normalized coupling $\kappa$ with the experimental feedback gain, we have to set $\kappa=k / 2$. The corresponding characteristic equation states

$$
\lambda=\lambda_{0}+\kappa\left(e^{-\lambda \tau}-1\right),
$$

which follows by applying the usual ansatz $z \sim e^{\lambda t}$ in Eq. (12). Control is in principle successful if $\operatorname{Re}\left(\lambda_{0}\right) \tau<2$ [15]. Within this constraint, the delay times for which control is most effective are odd multiples of $T_{0} / 2$, and control is not possible for even multiples of $T_{0} / 2$. If the fixed point is stabilized, the control force $\kappa\left(z_{\tau}-z\right)$ vanishes, so the control is noninvasive. A delay time modulation (or the use of distributed delays) will not change this feature, since at the fixed point $z_{\tau} \equiv z$ for all values of $\tau$, provided that the control is successful. Therefore, we can apply any delay modulation (distribution) and study its effect on the stability of the fixed point. Within our experimental constraints, we investigate three different types of variable (distributed) delay for control.

\section{A. Clock frequency modulation}

Delay modulation is created via modulation of the clock frequency, which drives the delay lines. All possible relations between $f(t)$ and $\tau(t)$ are given by Eq. (5). Figure 4 shows two examples, which satisfy this equation. Example (a) demonstrates the effect of a step function,

$$
f(t)=\left\{\begin{array}{l}
f_{1}, \quad t<t_{0}, \\
f_{2}, \quad t \geqslant t_{0},
\end{array}\right.
$$

with $f_{2}>f_{1}$. In this case, $\tau(t)$ follows a kink function, which at $t=t_{0}$ starts changing from the left constant value $\tau_{1}=N / f_{1}$ to the right constant value $\tau_{2}=N / f_{2}$. Both are trivial cases of a nonmodulated clock frequency. The duration of the transition is given by $\tau_{2}$, because this is the time that the buffer-which 
(a)

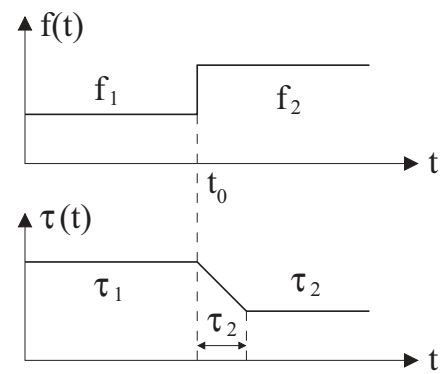

(b)

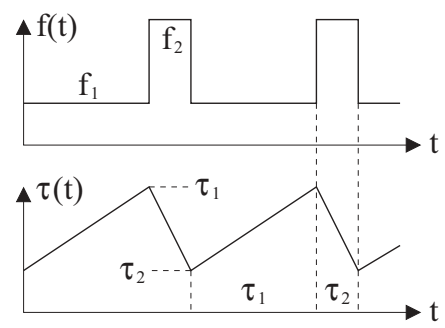

FIG. 4. Example relations between clock frequency modulation $f(t)$ and delay time modulation $\tau(t)$ after Eq. (5). (a) Effect of an instantaneous increase in clock frequency. (b) Construction of a sawtooth wave.

was previously recorded with rate $f_{1}$-needs to run through the FIFO with the new rate $f_{2}$.

Such a ramp is helpful in creating a triangle modulation in $\tau(t)$, which corresponds to a uniform delay distribution between two extremum values as in Eq. (8). The clock frequency modulation fulfilling the restrictions to create a triangle wave is an asymmetric rectangular wave with

$$
f(t)= \begin{cases}f_{1}, & \text { duration } \tau_{1}, \\ f_{2} & \text { duration } \tau_{2} .\end{cases}
$$

Here $T_{m}=\tau_{1}+\tau_{2}, \tau_{1}=N / f_{1}$, and $\tau_{2}=N / f_{2}$. Figure 4(b) illustrates the creation of such a modulation. If the intervals in $f(t)$ were not set correctly, the sawtooth in $\tau(t)$ would show clipping effects. Note that a similar modulation could also be achieved using larger values of $N$ with the same frequency modulation, because the triangular pattern reappears periodically by increasing $N$. But this also involves a higher mean delay time, which is not desirable, because the possible values are fixed by the choice of the modulation amplitude. So we restrict our setup to the "first Brillouin zone" in $N$.

Returning to our previous notation using $\tau_{0}$ and $\varepsilon$, we now aim to change those parameters independently. To this end, we use two delay lines serially. The first delay line is adjusted to a constant delay and the second line receives the described clock frequency modulation. The buffer sizes of both lines can be adjusted separately, so we have control over both mean delay time $\tau_{0}$ and modulation amplitude $\varepsilon$. Figure 5(a) shows the experimental setup for delay modulation. To keep the modulated clock frequency in a useful range, we choose $f_{v .1}=810 \mathrm{kHz}$ and $f_{v .2}=270 \mathrm{kHz}=f_{v .1} / 3$. For a given modulation amplitude $\varepsilon$, we then set the buffer size $N_{v}=f_{v .1} \varepsilon$, so that $\tau_{1}=\varepsilon$ and $\tau_{2}=3 \varepsilon$. The modulation period is $T_{m}=4 \varepsilon$. By construction, an offset of $\varepsilon$ in $\tau_{0}$ occurs additionally to the principal offset of $\varepsilon$ [because $\tau(t)$ cannot be negative]. Thus we can only examine the range $2 \varepsilon<\tau_{0}<2 \varepsilon+\tau_{c \text { max }}$. For
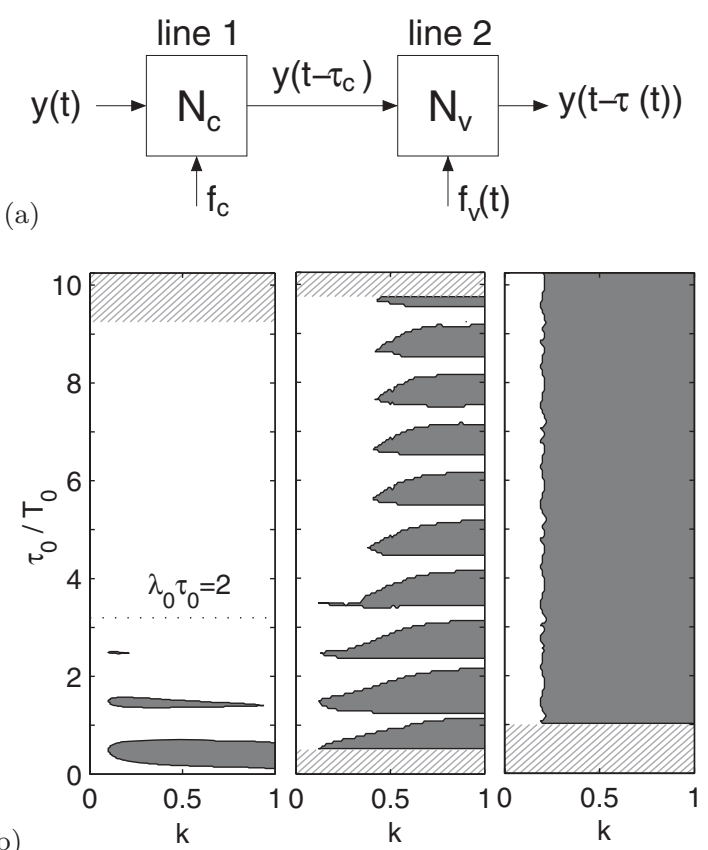

FIG. 5. Delay modulation for fixed point control. (a) Experimental setup with two delay lines for a modulation $\tau(t)$ between $\tau_{0}-\varepsilon$ and $\tau_{0}+\varepsilon$. (b) Scan of parameters $k$ and $\tau_{0}$ for different values of modulation amplitude $\varepsilon$. White: no control, oscillations with $\sigma_{y}>$ 0.1 V. Dark gray: control domains. Hatched areas mark parameter constellations that are not examined. Left: $2 \varepsilon=0$ (time-delayed feedback control). Middle: $2 \varepsilon=T_{0} / 2$ with $T_{m}=T_{0}$. Right: $2 \varepsilon=T_{0}$ and $T_{m}=2 T_{0}$. Oscillation period $T_{0}=2 \pi / \omega_{0}=0.63 \mathrm{~ms}$.

a fixed modulation amplitude, we scan the parameter space of feedback gain $k$ and mean delay time $\tau_{0}$ automatically by setting $N_{c}$ and $f_{c}$; see Fig. 5(a). For each constellation $\left(k, \tau_{0}\right)$, the oscillation strength of the circuit is measured in terms of the standard deviation $\sigma_{y}$ of the signal $y(t)$. If the oscillation is below some predefined value, here $y_{\mathrm{thr}}=0.1 \mathrm{~V}$, we define the dynamics as a stable fixed point. This threshold value is clearly larger than the noise level in the experiment $(10 \mathrm{mV})$ and significantly below the typical oscillation amplitudes of the uncontrolled oscillator (5 V); see Fig. 1. The results are a visualization of the control domains as shown in Fig. 5(b).

Without delay modulation $(\varepsilon=0)$ we recognize the stability islands, which are typical for TDFC. Stabilization of the fixed point is sensitive to the phase $\omega_{0} \tau$ and limited by $\operatorname{Re}\left(\lambda_{1 / 2}\right) \tau<2$, which in our case yields $\tau_{0}<3.2 T_{0}$. Here $T_{0}=$ $2 \pi / \omega_{0}=0.63 \mathrm{~ms}$ is the natural period of the uncontrolled oscillator. With increasing modulation amplitude, more and more of the parameter plane is covered by stable solutions, and the limitation in delay time is overcome. Setting the modulation amplitude equal to $\varepsilon=T_{0} / 2$ appears to have the strongest effect [right panel of Fig. 5(b)], although the increase of modulation amplitude has come along with a decrease of the modulation frequency. The results match the theoretical prediction for the distributed delay limit very well, so we conclude that the slow modulation does not significantly impede the mechanism, which has been found for high modulation frequencies. 


\section{B. Low-pass filter}

A rather trivial way to create a special delay distribution is the use of a low-pass filter. Let this filter be defined by

$$
\dot{w}=\beta[s(t)-w],
$$

in which $w \in \mathbb{R}$ and $\beta>0$. Practically, the filter is realized by an $R-C$ combination with the time constant $\beta=(R C)^{-1}$. The external signal $s(t)$ is low-pass filtered, which can be seen in the integral form of Eq. (16),

$$
w(t)=\int_{-\infty}^{t} s\left(t^{\prime}\right) \beta e^{-\beta\left(t-t^{\prime}\right)} d t^{\prime},
$$

which leads to the spectral power density

$$
S_{w}(f)=\frac{S_{s}(f)}{1+\frac{4 \pi^{2} f^{2}}{\beta^{2}}} .
$$

So if we low-pass filter the output of a delay line, before further constructing the control signal, the equations of motion of the controlled circuit become

$$
\begin{aligned}
\dot{x} & =\omega_{0}(-c x-y-z), \\
\dot{y} & =\omega_{0}[x+(a-c) y+k(w-y)], \\
\dot{z} & =\omega_{0}[g(x, z)-\gamma z], \\
\dot{w} & =\beta\left(y_{\tau_{0}}-w\right)
\end{aligned}
$$

and we realize a distributed delay feedback with the weight function

$$
\rho(\theta)= \begin{cases}0, & \theta<\tau_{0}, \\ \beta e^{-\beta\left(\theta-\tau_{0}\right)}, & \theta \geqslant \tau_{0} .\end{cases}
$$

Note that $\tau_{0}$ here is not the mean delay time of the distribution. The measurement is performed analogous to the previous one, here for different values of $\beta$, which is connected to the distribution width by $\beta \propto \varepsilon^{-1}$. Low values of $\beta$ correspond to a broad distribution due to a slowly reacting filter, and for $\beta \rightarrow \infty$ the filter simply transmits the signal of the delay line without change. The resulting control domains for this method are shown in Fig. 6, and we recognize a similar behavior as for
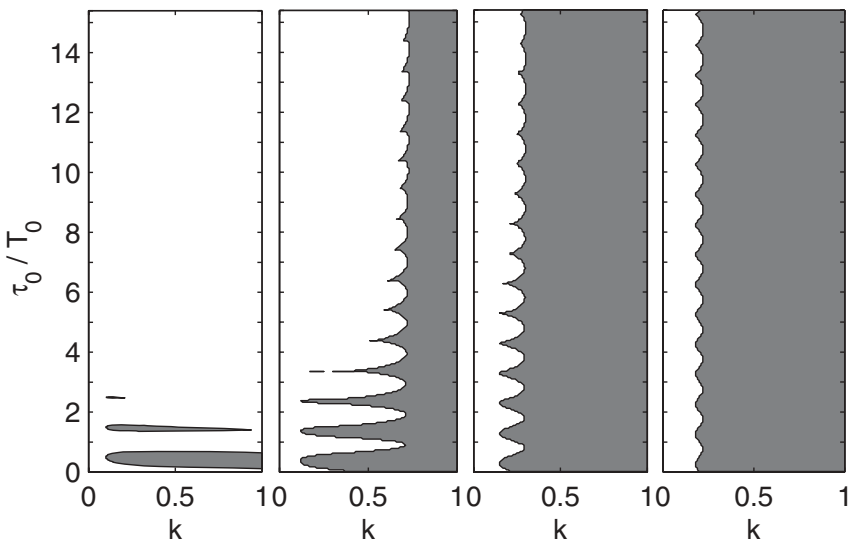

FIG. 6. Effect of a delay distribution created by a low-pass filter with filter constant $\beta$. The signal before filter has a delay $\tau_{0}$. Control domains $\left(\sigma_{y}<0.1 \mathrm{~V}\right)$ marked in dark gray. Left: $\beta \rightarrow \infty$, direct signal, TDFC. Left middle: $\beta=\omega_{0}$. Right middle: $\beta=\omega_{0} / 3$. Right: $\beta=\omega_{0} / 10$.

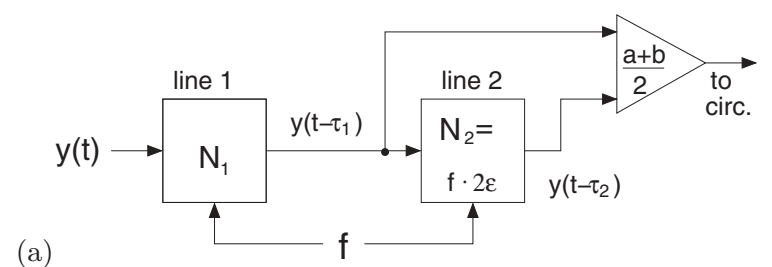

(a)
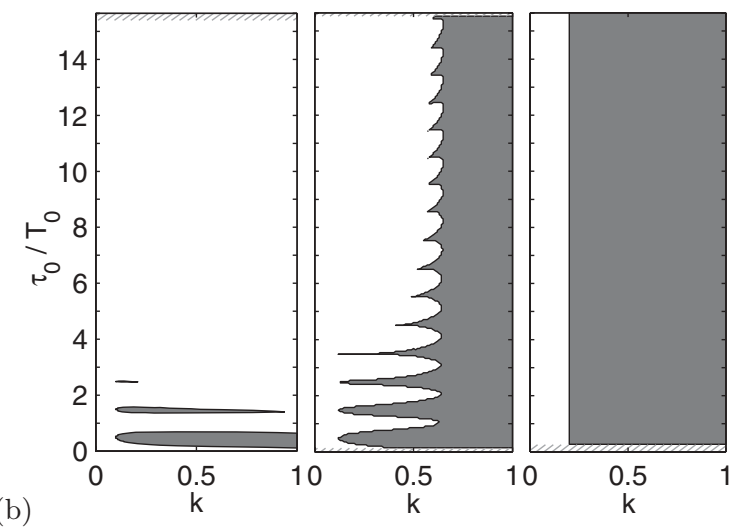

FIG. 7. Superposition of two delays. (a) Experimental setup. Delay of the first line is $\tau_{1}$. The delay of the second line is kept constant on $\tau_{2}-\tau_{1}=2 \varepsilon$. (b) Regions of fixed point control (dark gray, $\sigma_{y}<0.1 \mathrm{~V}$ ). Hatched areas are excluded from measurement. Left: $2 \varepsilon=0$ (time-delayed feedback control). Middle: $2 \varepsilon=T_{0} / 4$. Right: $2 \varepsilon=T_{0} / 2$.

the modulation case. For the broader distributions, we did not observe any limitation of the stabilization at large delay times.

\section{Two delays}

We repeat our parameter scan using two delay lines as shown in Fig. 7(a). The signals are superposed in such a way that the feedback force becomes

$$
k\left[\frac{1}{2}\left(y_{\tau_{1}}+y_{\tau_{2}}\right)-y\right]
$$

The resulting control is a realization of the multiple delay approach (MDFC) from Ahlborn and Parlitz [5,6]. The delay of the first line is $\tau_{1}=\tau_{0}-\varepsilon$ and the delay of the second line is $\tau_{2}=\tau_{0}+\varepsilon$. This setup corresponds to a symmetric square wave modulation of the delay time, in which the delay switches between $\tau_{1}$ and $\tau_{2}$ periodically. MDFC emerges as a special case of VDFC with rectangular modulations in the limit of high modulation frequency. We implemented MDFC as described by Eq. (21) in experiment, because fast switching between the two delays would have caused distortions, which can be avoided using two delay lines corresponding to the distributed delay limit of the modulation. The parameters $k$ and $\tau_{0}$ are again varied for some selected values of $\varepsilon$. Again, by the oscillation strength $\sigma_{y}$ of $y(t)$, we determine whether fixed point stabilization is possible. The results are shown in Fig. 7(b). They correspond largely to the previous findings and also integrate well in the context of MDFC. The optimal value of delay difference is $2 \varepsilon=T_{0} / 2$. For this case, also no limitation for large delays was observed. The possibility to control the unstable fixed point at arbitrarily large delay times $\tau_{0}$ is a striking feature, which all the investigated modulation and distribution techniques have in common. 


\section{Robustness of the control methods}

The robustness of the control methods is in principle already proven, because they withstand the intrinsic imperfections of our experiment. To further study this feature, we repeat selected measurements in the presence of additive noise. The signal of a noise generator is injected into the $y$ component of the circuit. The power spectrum of the generator covers a frequency band from $f_{\text {low }}=20 \mathrm{~Hz}$ to $f_{\text {high }}=40 \mathrm{kHz}(-3 \mathrm{~dB}$ bandwidth), so that it can be modeled by white noise. In the range that is relevant for the circuit, the spectral power density is $S(f)=\bar{S}=5 \mathrm{~V}^{2} / \mathrm{MHz}$ with an accuracy of $\pm 1 \mathrm{~dB}$. The equation for the $y$ component is then changed to

$$
\dot{y}=\omega_{0}\left[x+(a-c) y+k\left(y_{\tau}-y\right)\right]+\sqrt{2 D} \xi(t)
$$

with $\left\langle\xi(t) \xi\left(t^{\prime}\right)\right\rangle=\delta\left(t-t^{\prime}\right)$. The signal is injected via a $100 \mathrm{k} \Omega$ resistor instead of the standard $R_{4}=10 \mathrm{k} \Omega$, so that the noise intensity is rescaled by a factor 100 . Thus we obtain $2 D=$ $\omega_{0}^{2} \bar{S} / 100=5 \mathrm{~V}^{2} / \mathrm{s}$. We perform a parameter scan for each control method, in which the noise-induced oscillations in terms of the standard deviation $\sigma_{y}$ of $y(t)$ are measured. A low value corresponds to a high degree of stability and vice versa. To differentiate between very low values of $\sigma_{y}$, we evaluate the quantity

$$
\eta=\frac{D}{\sigma_{y}^{2}} .
$$

The choice of the definition for $\eta$ is motivated by analogy to an Ornstein-Uhlenbeck process with diffusion constant $D$, relaxation rate $\eta$, and variance $\sigma_{y}^{2}$. The results for our controlled fixed point are shown in Fig. 8. For all control techniques, there is no visible loss of robustness at large delay times. This result is even more peculiar, since from numerical simulations we have seen that the maximum exponent of the controlled system tends toward zero for large delays; see Sec. VI. We also investigated this issue in experiment. By switching on the control force $k$ at some time $t_{0}$, one can

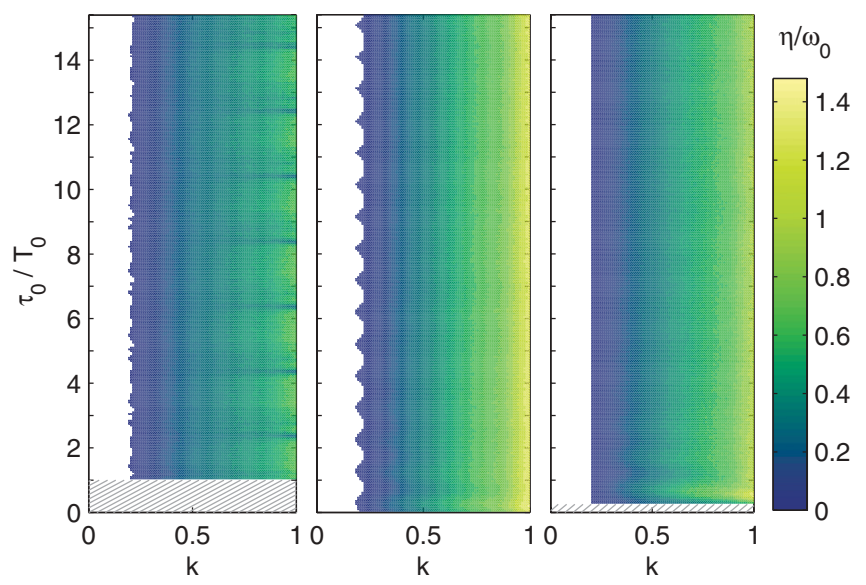

FIG. 8. (Color online) Parameter scans with additive noise. White: no control $\left(\sigma_{y}>0.1 \mathrm{~V}\right)$. Colors correspond to $\eta\left(k, \tau_{0}\right)$ in the control domains. Hatched: not measured. Left: control by variabledelay feedback with $2 \varepsilon=T_{0}$ and $T_{m}=2 T_{0}$. Middle: low-pass filtered feedback with $\beta=\omega_{0} / 10$. Right: two-delay feedback with $2 \varepsilon=$ $T_{0} / 2$.
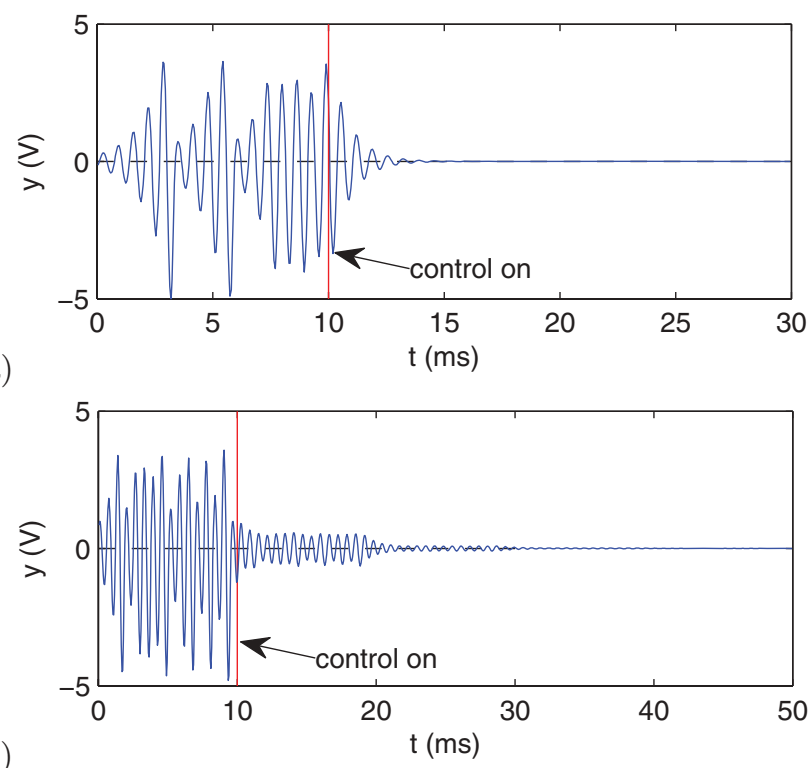

(b)

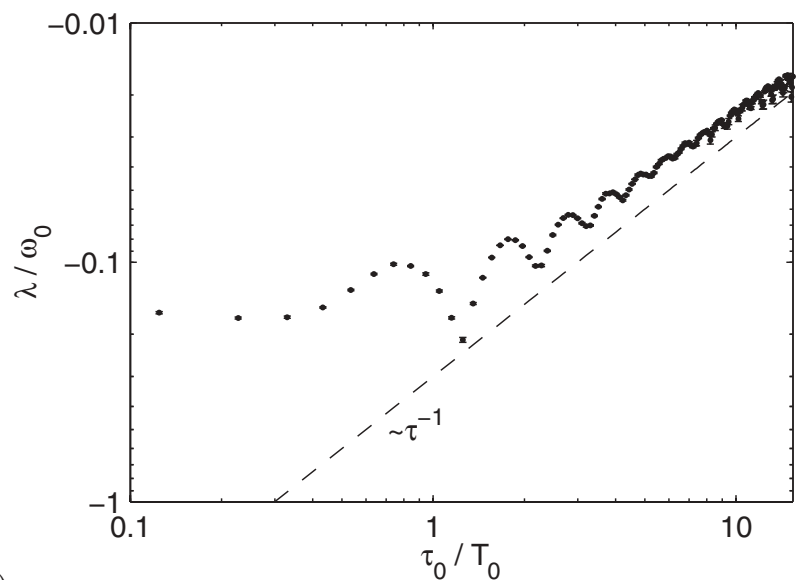

FIG. 9. (Color online) Analysis of transients for low-pass filtered feedback control. (a) Transient at $\tau_{0}=T_{0}$. (b) Transient at $\tau_{0}=14 T_{0}$. (c) Power-law dependence of the stability exponent $\lambda$ on the delay $\tau_{0}$.

observe transients and determine the relaxation rate toward the stable fixed point. This has been done for the low-pass filter control method at different delay times. Figure 9 shows some typical transients, which appear after the onset of control for small and large delay times $\tau_{0}$. The evaluation of the decay rate $\lambda\left(\tau_{0}\right)$ for all transients also reveals a power law, which would be typical in the presence of a pseudocontinuous spectrum; see, e.g., [16]. In summary, these findings clearly show that the presented control methods work well for large delay times, although the leading exponent is of order $O\left(1 / \tau_{0}\right)$, which at first glance is counterintuitive. But the reaction to noise takes into account more of the stable modes (see, e.g., [17]), which then leads to the $\tau_{0}$-independent behavior.

\section{STABILIZATION OF PERIODIC ORBITS}

Variable or distributed delay feedback is also suitable for the control of unstable periodic orbits. In contrast to fixed point control, here one cannot apply an arbitrary modulation, because delays which differ from multiples of the orbit period 
will lead to an invasive feedback force. Any delay modulation is allowed, which in the limit of high modulation frequency creates a distribution

$$
\rho(\theta)=\sum_{n=1}^{\infty} a_{n} \delta\left(\theta-n T_{p}\right),
$$

where $T_{p}$ is the period of the orbit and $\sum_{n} a_{n}=1$. Note that in this general notation, also the case of extended TDFC is included $[3,18,19]$. For ETDFC, the coefficients have to be chosen as $a_{n}=(1-R) R^{n-1}$, where $R$ is the recursion factor of the geometric sum. $R \rightarrow 0$ gives $a_{n}=\delta_{n, 1}$, which corresponds to TDFC. A value of $R$ close to 1 or -1 takes into account many multiples of the delay time.

\section{A. Two-delay control}

In this paper, we want to focus on those types of modulation (distribution) that create contributions to the sum in a not very large interval of delay times. The simplest case is given by the superposition of two delays similar to Eq. (21). To show the theoretically predicted performance improvement in experiment, we choose to control the unstable period-1 orbit of our chaotic circuit, which has a period $T_{p} \approx T_{0}=$ $2 \pi / \omega_{0}$. The instability of this orbit increases monotonically with the main control parameter $a$, so we study the stabilization of the orbit in the $k a$ parameter plane. Analogous to the automatic scan technique used for the fixed point, we measure the deviation between $y(t)$ and $y\left(t-T_{p}\right)$ to determine whether the orbit is successfully controlled. We want to compare our two-delay control technique to ordinary TDFC in a regime where the classical method largely fails. This can be achieved by setting a large delay time, because an orbit can only be stabilized if $\tau<2 / \operatorname{Re}\left(\Lambda_{0}\right)$, where $\Lambda_{0}$ is the Floquet exponent of the uncontrolled orbit. The claim for noninvasiveness and the torsion of the orbit restrict the possible values of $\tau$ to odd multiples of $T_{p}$. So we set for TDFC a delay time $\tau=$ $3 T_{p}$, for which the general limit of stability is more easily exceeded than for the standard setting $\tau=T_{p}$. As a suitable example of distributed delay control, we take a superposition of two different delayed signals with delays $\tau_{1}=3 T_{p}$ and $\tau_{2}=$ $4 T_{p}$. The results are shown in Fig. 10 and clearly demonstrate the improvement of stabilization. Additionally, we repeat the experiment using the extended TDFC method. Here a single delay line is also set to $\tau=3 T_{p}$, as for the TDFC measurement.

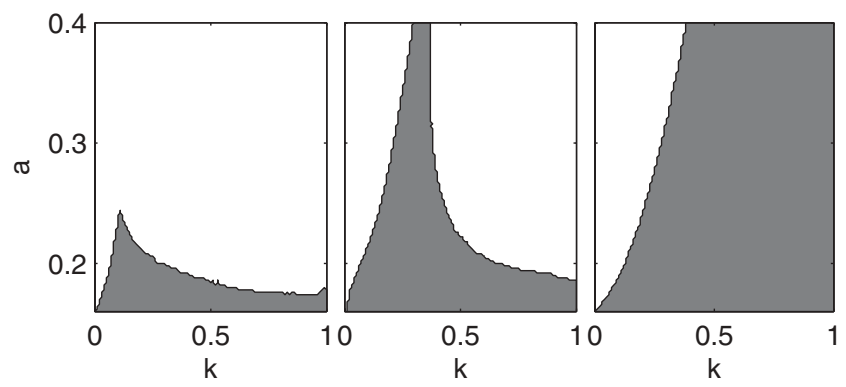

FIG. 10. Scan of the $a k$ parameter plane for control of period-1 orbit; see Fig. 1, top. Control domains [standard deviation of $y(t-$ $\left.T_{p}\right)-y(t)$ is $\left.<0.1 \mathrm{~V}\right]$ are marked in gray. Left: TDFC. Middle: ETDFC with $R=0.5$. Right: two-delay distributed delay feedback.
But instead of receiving only the direct signal $y(t)$, the delay line receives a sum of $y(t)$ and its own output, which leads to the geometric sum of multiple delays. Direct input and recursive input are equally weighted, so we obtain a recursion factor $R=0.5$. The resulting control domain is included in Fig. 10 and demonstrates improvement compared to simple TDFC. However, the control interval narrows for increasing instability, which in the examined range was not observed for the two-delay control method.

\section{B. Finite modulation frequency}

The direct superposition of two delays as presented above represents the distributed delay limit of fast switching between the delays $\tau_{1}$ and $\tau_{2}$. Beyond this, we aim to address two additional questions: (i) Which modulation frequencies in variable-delay control are high enough to obtain the same results as for distributed delays? (ii) It has been shown theoretically that a special choice of the modulation frequency leads to a further improvement compared to distributed delays. Is this phenomenon observable in experiment?

To clarify this, we construct a setup similar to the previous one, but with the possibility to modulate the weights of each delay line using a variable gain. The gain is implemented by multipliers from type AD633. We could also have built a setup in which we switch between the delay lines. But it turned out that at the moment of switching, high-frequency distortions are generated, which spoil the noninvasiveness of the control and thus make it hard to determine when the orbit has been controlled successfully. To avoid distortions arising from very high-frequency components, we modulate between the delay lines sinusoidally, so that the control force becomes

$$
k\left\{\mu(t) y_{\tau .1}+[1-\mu(t)] y_{\tau .2}-y\right\}
$$

where $\mu(t)=\frac{1}{2}\left[1+\sin \left(2 \pi f_{m} t\right)\right]$ is the modulation signal. We are interested in the frequency dependence of the control boundaries $k_{\min }$ and $k_{\max }$. Without considerable distortions, we can only apply a coupling gain $k \in[0,1]$. But similar to the case of two-delay control as shown in Fig. 10, we come across the problem that $k_{\max }>1$ for standard parameters. However, an increase of the parameter $a$ in our circuit leads to orbits with a higher degree of instability, for which the control interval should become narrow enough to be observed completely. At least for $a=0.5$ we found the control boundaries of the period-1 orbit to lie in a detectable range. For this orbit, we measure the strength $\sigma\left(k, f_{m}\right)$ of the control signal before gain, which vanishes in the case of successful stabilization. The detected control domain for modulation between $\tau_{1}=1 T_{p}$ and $\tau_{2}=2 T_{p}$ is shown in Fig. 11 .

In general, one can see that the dependence on a finite modulation frequency is not very strong, meaning that-as found for the fixed point-variable delay works about as well as the corresponding distributed delay. The transition to the distributed delay limit can be seen in the regime about one order of magnitude above the main oscillation frequency $f_{p}$. Below, there are interactions between delay modulation and frequency components of the oscillator, which in their complete appearance are nontrivial. The only phenomenon, which already has been theoretically explained, is the enhancement of stabilization at half the orbit frequency and 

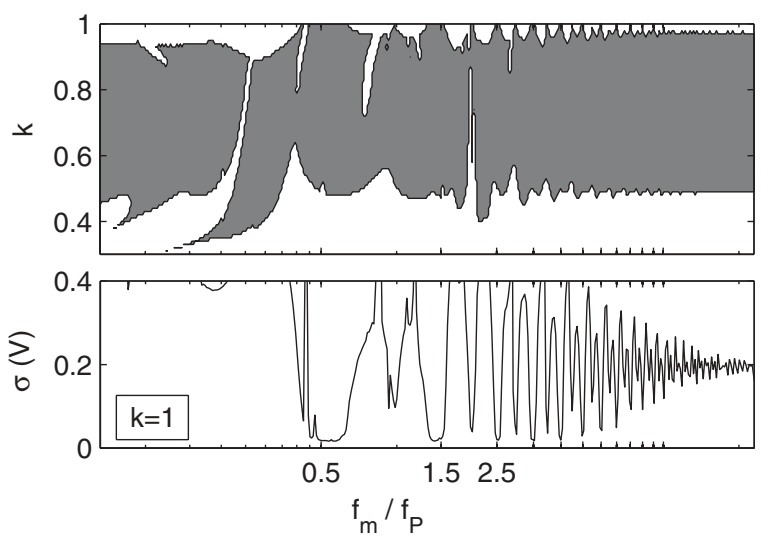

FIG. 11. Control of $\mathrm{P} 1$ orbit at $a=0.5$ with $f_{p}=T_{p}^{-1}=$ $1.51 \mathrm{kHz}$. Delay modulation between $\tau_{1}=T_{p}$ and $\tau_{2}=2 T_{p}$ with frequency $f_{m}$. Top: stabilization domain (dark gray, $\sigma<0.1 \mathrm{~V}$ ) in dependence of feedback gain $k$ and modulation frequency. Bottom: strength $\sigma$ of remaining control signal at $k=1$ showing optimal control at odd multiples of $f_{p} / 2$. Displayed frequency range: $100 \mathrm{~Hz}-$ $40 \mathrm{kHz}$.

odd multiples of it. The control domain recorded in experiment is extended toward larger values of $k$, which confirms the theoretical prediction very well. It is also remarkable that the resonance phenomena are present in both experiment and theory, although the types of modulation were very differentsinusoidally sloshing distribution in experiment and periodic switching in simulation. This demonstrates a high robustness of the control mechanism against variations in the shape of the modulation.

\section{MECHANISM OF STABILIZATION}

Stability analysis for the presented modulated (distributed) feedback technique can be regarded as mostly covered by our previous theoretical work as well as others [5,8-10,20]. For completeness, we give here an interpretation as well as exemplifying analytical and numerical calculations for the mechanism behind the observed phenomena. Essentially it can be discussed in terms of a normal form,

$$
\dot{z}(t)=\left(\alpha_{0}+i \omega_{0}\right) z(t)+\kappa\{z[t-\tau(t)]-z(t)\},
$$

with $\alpha_{0}>0$ and $\omega_{0}, \kappa \in \mathbb{R}$. In the distributed-delay limit, this equation becomes

$$
\dot{z}(t)=\left(\alpha_{0}+i \omega_{0}\right) z(t)+\kappa\left(\int_{0}^{\infty} \rho(\theta) z(t-\theta) d \theta-z(t)\right) .
$$

With an exponential ansatz, we obtain the eigenvalue equation

$$
\lambda=\alpha_{0}+i \omega_{0}+\kappa\left(\int_{0}^{\infty} \rho(\theta) e^{-\lambda \theta} d \theta-1\right) .
$$

If $\rho(\theta)$ is nonzero only between $\tau_{0}-\varepsilon$ and $\tau_{0}+\varepsilon$, then we can refine the above expression further to

$$
\begin{aligned}
\lambda & =\alpha_{0}+i \omega_{0}+\kappa\left(e^{-\lambda \tau_{0}} \int_{-\varepsilon}^{+\varepsilon} \rho\left(\tau_{0}+\theta\right) e^{-\lambda \theta} d \theta-1\right) \\
& =\alpha_{0}+i \omega_{0}+\kappa\left[e^{-\lambda \tau_{0}} \chi(\lambda, \varepsilon)-1\right] .
\end{aligned}
$$

Here the quantity $\chi(\lambda, \varepsilon)$ summarizes the effect of a given modulation (distribution). In the nonmodulated case, we have simply $\chi(\lambda, 0) \equiv 1$, which then reveals the known characteristic equation for TDFC. Given Eq. (29), it is easy to imagine a scenario in which improved stabilization is obvious: If one could find a distribution $\rho(\theta)$ such that $\chi(\lambda, \varepsilon)=0$, then Eq. (29) would reduce to

$$
\lambda=\alpha_{0}+i \omega_{0}-\kappa .
$$

Here for $\kappa>\alpha_{0}$ the fixed point would be stable, regardless of how large the delay time is. In practice, it is difficult to keep $\chi \equiv 0$, but the desired effect already appears to be strong enough if $\chi$ is only close to zero. By the following examples, we show how the presented modulation (distribution) techniques satisfy this criterion of effectively reducing the weight of the delay term in Eq. (29).

\section{A. Numerical examples: Delay modulation}

A fast triangular modulation between two extremal values of the delay time, $\tau_{1}=\tau_{0}-\varepsilon$ and $\tau_{2}=\tau_{0}+\varepsilon$, is equivalent to a uniform delay distribution

$$
\rho(\theta)= \begin{cases}1 /(2 \varepsilon), & \tau_{1} \leqslant \theta \leqslant \tau_{2}, \\ 0, & \text { otherwise. }\end{cases}
$$

This leads to

$$
\chi(\lambda, \varepsilon)=\frac{\sinh (\lambda \varepsilon)}{\lambda \varepsilon} .
$$

Equation (29) then becomes

$$
\lambda-\alpha_{0}-i \omega_{0}+\kappa\left[1-e^{-\lambda \tau_{0}} \sinh (\lambda \varepsilon) /(\lambda \varepsilon)\right]=0 .
$$

Numerical calculation of the stability domain from the characteristic Eq. (33) gives the diagram shown in Fig. 12. Panels (a)-(d) correspond to different values of the amplitude parameter $\varepsilon$ normalized by the intrinsic period $T_{0}=2 \pi$ of the uncontrolled system: (a) $\varepsilon / T_{0}=0.125$, (b) $\varepsilon / T_{0}=0.25$, (c) $\varepsilon / T_{0}=0.5$, (d) $\varepsilon / T_{0}=1$. The parameters of the unstable spiral are $\alpha_{0}=0.1$ and $\omega_{0}=1$. The color codes at the right end of the panel denote the values of the largest real part of the complex eigenvalues obtained numerically from Eq. (33). The shaded region in each panel denotes the negative values of the maximum real part of the complex eigenvalues, i.e., it depicts only the control parameters for which the stabilization is successful.

As is seen from panels (a)-(d), an increase of the modulation amplitude $\varepsilon / T_{0}$ leads to a larger control domain. Further numerical analysis shows that in this case the increase of the stability area is monotonic upon increasing the value of $\varepsilon / T_{0}$. To illustrate the superiority of the control method with respect to the standard delayed feedback control schemes, in Fig. 13 we show numerically calculated control domains in the case of TDFC [panel (a)] and ETDFC [panel (b)] for the same parameters of the uncontrolled system. In the case of ETDFC, the memory parameter is chosen as $R=0.5$.

\section{B. Numerical examples: Two delays}

Fast and symmetric switching between two delay times $\tau_{1}=\tau_{0}-\varepsilon$ and $\tau_{2}=\tau_{0}+\varepsilon$ (i.e., square-wave modulation) is 

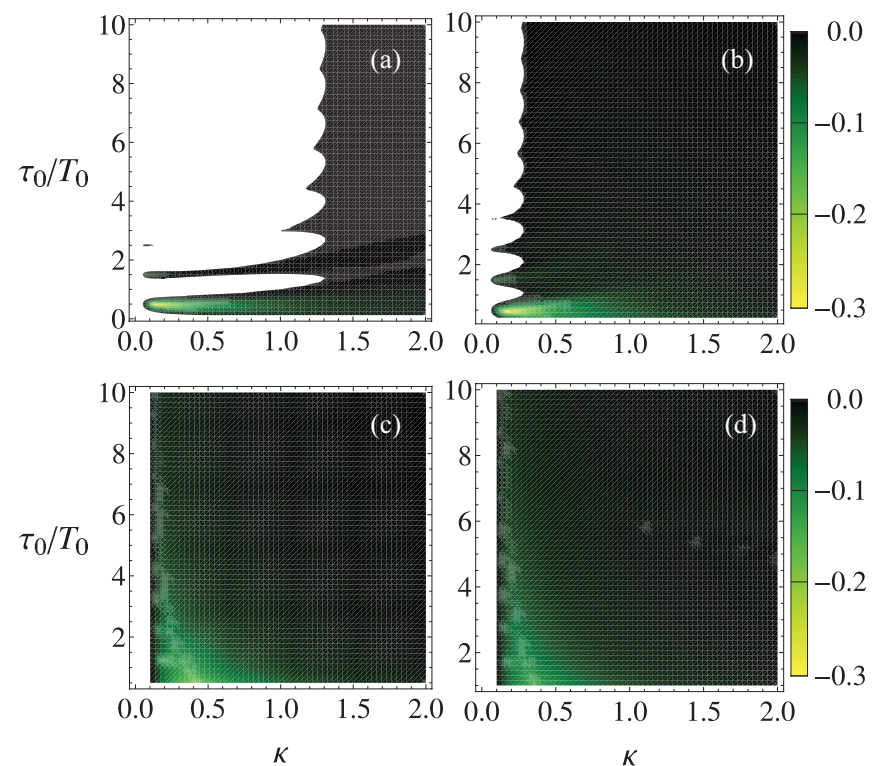

FIG. 12. (Color online) Stability domains of the fixed point $z=0$ in the plane parametrized by the feedback gain $\kappa$ and the nominal time delay $\tau_{0} / T_{0}$ for a triangular modulation of the delay and different modulation amplitudes $\varepsilon / T_{0}$ : (a) $\varepsilon / T_{0}=0.125$, (b) $\varepsilon / T_{0}=0.25$, (c) $\varepsilon / T_{0}=0.5$, (d) $\varepsilon / T_{0}=1$. White: no control. Colors correspond to the real part of $\lambda$ after Eq. (33).

equivalent to a two-peak delay distribution

$$
\rho(\theta)=\frac{1}{2}\left[\delta\left(\theta-\tau_{0}+\varepsilon\right)+\delta\left(\theta-\tau_{0}-\varepsilon\right)\right] .
$$

According to Eq. (29), we obtain

$$
\chi(\lambda, \varepsilon)=\cosh (\lambda \varepsilon) .
$$

In this case, we arrive at the quasipolynomial equation

$$
\lambda-\alpha_{0}-i \omega_{0}+\kappa\left[1-e^{-\lambda \tau_{0}} \cosh (\lambda \varepsilon)\right]=0 .
$$

In Fig. 14, we give the numerically calculated control domains by using Eq. (36). Contrary to the observations for the domain enlargement in the case of a triangular-wave modulation, here the variation of the stability area is nonmonotonic upon increasing the modulation amplitude. The control domain increases its area until a certain value of the modulation amplitude, after which the area is decreased and the stability islands are rearranged. With further increase of the amplitude $\varepsilon$, the
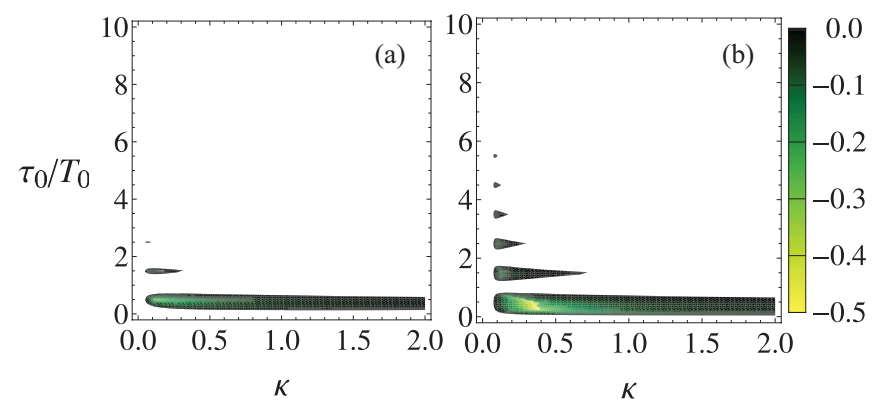

FIG. 13. (Color online) Stability domains in the absence of delay modulation: (a) Pyragas' time-delayed feedback control (TDFC), (b) extended time-delayed feedback control (ETDFC) due to Socolar et al. [3] with $R=0.5$. White: no control. Colors correspond to the real part of $\lambda$ from Eq. (29).
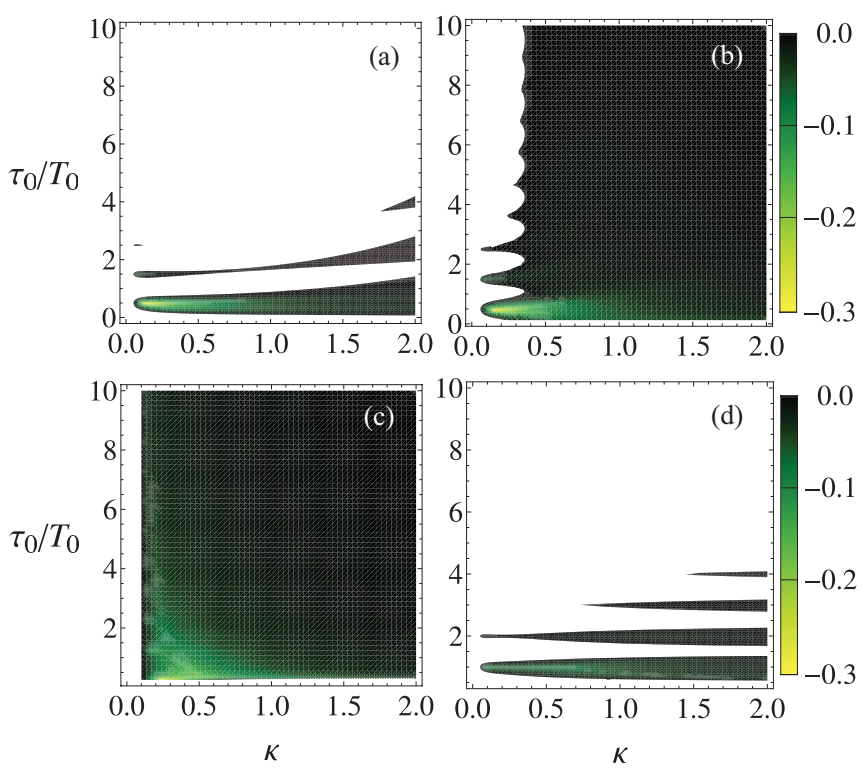

FIG. 14. (Color online) Stability domains for the controlled origin in the parametric plane spanned by the feedback gain $\kappa$ and the nominal delay $\tau_{0} / T_{0}$ for a square-wave modulation of the delay. The modulation amplitudes are (a) $\varepsilon / T_{0}=0.0625$, (b) $\varepsilon / T_{0}=0.125$, (c) $\varepsilon / T_{0}=0.25$, and (d) $\varepsilon / T_{0}=0.5$. White: no control. Colors correspond to the real part of $\lambda$ after Eq. (36).

stability area is increasing again, and this alternating behavior of increasing and decreasing the area of stabilization continues. This effect could also be reproduced in experiment. In contrast, for the modulation case with increasing modulation amplitude the control performance gets worse in experiment, contrary to the theoretical expectation. This is explained by the fact that in our modulation setup, the modulation period $T_{m}$ is also proportional to the modulation amplitude $\varepsilon$ by construction. So the failure of control for large values of $\varepsilon$ can be related to the low modulation frequency. The numerical results obtained by the distributed-delay approximation are no longer valid in this case.

\section{Interpretation}

From the above examples, we get an understanding of the basic mechanism behind variable (distributed) delay feedback control. Having a closer look at the boundaries of control, where $\operatorname{Re}(\lambda)=0$, we see that the expression for the coefficient $\chi$ reduces to

$$
\chi_{a}=\frac{\sin (\omega \varepsilon)}{\omega \varepsilon}
$$

for the constant distribution in example (a), and

$$
\chi_{b}=\cos (\omega \varepsilon)
$$

for the two-peak distribution in example (b). These are the Fourier transforms of the corresponding delay distributions, which can also be seen from generating Eq. (29) if $\lambda$ is set to $i \omega$. So at the threshold to stability, the distribution in (a) acts like a "single slit" and the distribution in (b) acts like a "double slit," respectively. Optimal control can in these terms be found for those values of $\varepsilon$ which create complete destructive "interference," i.e., $\chi=0$. Numerical 
calculations for our setup show that $\omega \approx \omega_{0}=2 \pi / T_{0}$ is a valid approximation, so that we find optimal control at $\varepsilon=n T_{0} / 2$ for example (a), and $\varepsilon=(n+1 / 2) T_{0} / 2$ for example (b), which is in excellent agreement with the experimental findings.

\section{CONCLUSIONS}

We have shown that the use of variable (distributed) delays in a time-delayed feedback control scheme can significantly improve the control performance for unstable fixed points as well as for unstable periodic orbits. We have proven the robustness and reliability of our control method by an experiment with a chaotic electronic oscillator and digital delay lines. The autonomous diode oscillator has an unstable fixed point, which is susceptible for control by the Pyragas method and its modifications, as well as a set of periodic orbits, which are amenable to control by the same methods.

The fixed point allows for any delay modulation (distribution), from which we realized three different types: (i) modulation of the delay time via modulation of the clock frequency driving the delay lines, (ii) an exponential delay distribution using one delay line and a low-pass filter, and (iii) a superposition of two equally weighted delays using two parallel delay lines. The effect of all variations is comparable: New domains of control are generated at large values of delay time and coupling strength. For an optimal value of the modulation amplitude (distribution width), we obtain one connected domain of control, which extends to arbitrarily large delay times. We were able to show that for large mean delay, control works in a robust way in spite of the decreasing magnitude of the leading stability exponent.

Periodic orbits allow for such a delay modulation (distribution), in which only multiples of the orbit period are included. Here we were able to show that switching between two delays with a difference of a single period of the orbit leads to similar extensions of the control domain as observed for the fixed point. Furthermore, we were able to stabilize orbits which cannot be stabilized by TDFC. As for the fixed point, a finite modulation frequency does not disturb the mechanism. In contrast, if the modulation frequency matches odd multiples of half the orbit frequency, we found the stabilization to be optimal.

The mechanism of our control method can be explained in terms of destructive interference. The intrinsic torsion of an orbit or fixed point, which is necessary for TDFC to work in principle, is used by the delay modulation (distribution) in such a way that different phases of the torsion cancel each other out. This leads to minimization of the effect of the delay term, which in most cases is an undesirable source of instability, especially at large delays.
[1] K. Pyragas, Phys. Lett. A 170, 421 (1992).

[2] Handbook of Chaos Control, 2nd ed., edited by E. Schöll and H. G. Schuster (Wiley-VCH, Weinheim, 2008).

[3] J. E. S. Socolar, D. W. Sukow, and D. J. Gauthier, Phys. Rev. E 50, 3245 (1994).

[4] J. E. S. Socolar and D. J. Gauthier, Phys. Rev. E 57, 6589 (1998).

[5] A. Ahlborn and U. Parlitz, Phys. Rev. Lett. 93, 264101 (2004).

[6] A. Ahlborn and U. Parlitz, Phys. Rev. E 72, 016206 (2005).

[7] A. Gjurchinovski and V. Urumov, Europhys. Lett. 84, 40013 (2008).

[8] F. M. Atay, Phys. Rev. Lett. 91, 094101 (2003).

[9] K. Konishi, H. Kokame, and N. Hara, Phys. Lett. A 374, 733 (2010).

[10] Y. Kyrychko, K. Blyuss, and E. Schöll, Eur. Phys. J. B 84, 307 (2011).

[11] T. Jüngling, Ph.D. thesis, TU Darmstadt, 2010.
[12] T. Jüngling, H. Benner, H. Shirahama, and K. Fukushima, Phys. Rev. E 84, 056208 (2011).

[13] S. Heiligenthal, T. Dahms, S. Yanchuk, T. Jüngling, V. Flunkert, I. Kanter, E. Schöll, and W. Kinzel, Phys. Rev. Lett. 107, 234102 (2011).

[14] W. Michiels, V. Van Assche, and S.-I. Niculescu, IEEE Trans. Autom. Control 50, 493 (2005).

[15] W. Just, E. Reibold, H. Benner, K. Kacperski, P. Fronczak, and J. Hołyst, Phys. Lett. A 254, 158 (1999).

[16] S. Yanchuk and P. Perlikowski, Phys. Rev. E 79, 046221 (2009).

[17] D. Hunt, G. Korniss, and B. K. Szymanski, Phys. Rev. Lett. 105, 068701 (2010).

[18] K. Pyragas, Phys. Lett. A 206, 323 (1995).

[19] M. Bleich and J. Socolar, Phys. Lett. A 210, 87 (1996).

[20] A. Ahlborn and U. Parlitz, Phys. Rev. Lett. 96, 034102 (2006). 